\title{
Glucocorticoid treatment, immobility, and constipation are associated with nutritional risk
}

\author{
Jean-Pierre Gutzwiller • Josef Aschwanden • \\ Samuel Iff • Michèle Leuenberger • Martin Perrig • \\ Zeno Stanga
}

Received: 26 July 2010/Accepted: 15 February 2011/Published online: 3 March 2011

(C) Springer-Verlag 2011

\begin{abstract}
Purpose The hypothesis of this clinical study was to determine whether glucocorticoid use and immobility were associated with in-hospital nutritional risk.

Methods One hundred and one patients consecutively admitted to the medical wards were enrolled. Current medical conditions, symptoms, medical history, eating and drinking habits, diagnosis, laboratory findings, medications, and anthropometrics were recorded. The Nutrition Risk Score 2002 (NRS-2002) was used as a screening instrument to identify nutritional risk.

Results The results confirmed that glucocorticoid use and immobility are independently associated with nutritional risk determined by the NRS-2002. Constipation could be determined as an additional cofactor independently associated with nutritional risk.

Conclusions Glucocorticoid treatment, immobility, and constipation are associated with nutritional risk in a mixed hospitalized population. The presence of long-time
\end{abstract}

J.-P. Gutzwiller · S. Iff · M. Leuenberger · Z. Stanga Division of Endocrinology, Diabetes and Clinical Nutrition, Bern University Hospital and University of Bern,

Bern, Switzerland

J.-P. Gutzwiller ( $\square)$

Division of Gastroenterology, Hospital Zofingen,

Mühlethalstrasse 27, 4800 Zofingen, Switzerland

e-mail: jean-pierre.gutzwiller@unibas.ch

J. Aschwanden $\cdot$ M. Perrig $\cdot$ Z. Stanga

Department of General Internal Medicine, Bern University

Hospital and University of Bern, Bern, Switzerland

S. Iff

Division of Clinical Epidemiology and Biostatistics,

Institute of Social and Preventive Medicine,

University of Bern, Bern, Switzerland glucocorticoid use, immobility, or constipation should alert the clinician to check for nutritional status, which is an important factor in mortality and morbidity.

Keywords Malnutrition - Nutritional risk screening · Glucocorticoid treatment $\cdot$ Immobility $\cdot$ Constipation

\section{Introduction}

Protein-energy malnutrition (PEM) is an independent risk factor for increased number of complications, increased morbidity and mortality, increased length of hospital stay, and increased costs [1-3] and has a prevalence of between 30 and 50\% in hospitalized patients [4-6]. Malnutrition and illness influence each other: on the one hand, the illness itself can worsen the nutritional state, and on the other hand, malnutrition can negatively affect the course of the illness. As malnutrition is a largely treatable co-morbidity, rapid and simple identification and effective management of this condition are essential [7].

For hospitalized patients, the NRS-2002, introduced by Kondrup et al., is a valid and reliable indicator of patients at nutritional risk [8]. Nutritional risk includes characteristics that are associated with an increased likelihood of poor nutritional status. The purpose of nutritional screening is to predict the probability of a better or worse outcome due to nutritional factors and whether nutritional treatment is likely to influence this. Our group has shown that patients at nutritional risk undergoing elective colorectal surgery have higher in-hospital mortality and morbidity [9]. However, mechanisms and cofactors involved and related to malnutrition have not been identified.

Sarcopenia is defined as age- or disease-related muscle loss, and nutritional risk is very well known in geriatric 
patients. A study from the group of Sieber and co-workers presented a model for the connections between frailty, sarcopenia, and risk of malnutrition in geriatric patients [10]. The authors described a vicious cycle involving sarcopenia and immobilization: sarcopenia leads to neuromuscular impairment, resulting in falls and fractures. The consequence of fractures is immobilization, which ultimately induces sarcopenia.

PEM is believed to cause hypercortisolemia [11]. Cortisol's effects are mediated through the glucocorticoid receptor, which binds the hormone in the cytosol, translocates to the nucleus, and promotes gene transcription [12]. Lunn and co-workers found a dose-dependent increase in the plasma concentration of cortisol in relation to muscle wasting in children: the more wasted the children's muscles, the higher the cortisol levels observed [13]. In rat experiments, injection of glucocorticoids led to a slow, continuous decrease in body weight throughout the experiment. Analysis of the weight loss showed that glucocorticoid-treated animals lost more muscle mass than controls. Interestingly, the glucocorticoid-treated rats did not have any change in food intake compared to controls [14]. Glucocorticoids not only stimulate proteolysis but also inhibit protein synthesis and the transport of amino acids into muscle, and these coordinated actions promote the mobilization of amino acids used for glucogenesis [15]. Hypercortisolism has been implicated as an important contributing factor in muscle wasting: in vitro experiments have shown induction of muscle catabolism by glucocorticoids [16]. An interesting model for glucocorticoid excess in humans is Cushing's disease. Weakness and proximal muscle wasting are common in Cushing's syndrome and are induced by the catabolic effects of excess glucocorticoids on skeletal muscle. The catabolic effects of glucocorticoids are amplified by physical inactivity [17]. Nevertheless, other researchers hypothesize that pure glucocorticoid excess does not cause weight loss but rather weight gain and visceral obesity and that normalization of glucocorticoid excess in Cushing's syndrome is associated with significant loss of body weight, but muscle mass remained on a constant low level [18]. In addition, extended use of glucocorticoids in patients with inflammatory bowel disease is associated with mortality in contrast to the use of other immunosuppressants [19].

A period of confinement in bed often precedes death [20]. Although immobility is a key aspect of sarcopenia, we found few reports on this issue in the medical literature. This is surprising, because hospital patients are frequently bedridden. In addition, constipation has been associated with a bedridden state in geriatric patients [21, 22].

Our hypothesis was that glucocorticoid use and immobility are associated with in-hospital nutritional risk and that constipation can act as a modifier in this context.

\section{Materials and methods}

Study design

In a cross-sectional study of patients hospitalized on the general internal medicine wards of the Bern University Hospital, we investigated possible factors for nutritional risk as defined by the NRS-2002.

Selection of participants, recruitment, and approval

Over four consecutive weeks, all patients admitted to the wards were invited to participate in the study. Patients were considered eligible if they were 18 years or older and were willing and able to give written informed consent. Exclusion criteria were patients with psychological disorders, dementia, poor knowledge of the study language, or symptomatic heart, renal, or liver failure. All participants provided written informed consent before enrollment, and the study protocol was approved by the ethics committee of the University of Bern.

\section{Data and measurements}

A specialist in clinical nutrition (trained physician) screened the participants for nutritional risk using the NRS-2002 [23] within $24 \mathrm{~h}$ after admission. Nutritional risk was evaluated by two components-nutritional state and severity of disease-each with a value ranging from 0 to 3 points. The score of severity of disease reflects increased nutritional requirements due to stress metabolism. Nutritional state was based on three variables: body mass index (BMI), recent weight loss, and dietary intake during the last week before admission. The most compromised of the three variables is used for the NRS-2002 to categorize the patient. Dietary intake, as a semi-quantitative scale, was included and was given the same weight as the other variables, despite the fact that few previous studies have used dietary history as part of the initial characterization of the patients. Classification of a patient as at risk using dietary history alone will only occur with $a \geq 1$ week's history of an intake equal to $0-25 \%$ of a patient's estimated nutritional requirements, and such a history can probably be obtained with a sufficient degree of certainty in most cases. It has been shown that nurses' dietary recording in quartiles agrees reasonably well with the dietician's recording [24], and it may be assumed that a recent dietary history also will be reasonably reliable.

The score for nutritional status was added to the score for severity of disease to give a total score, which could range from 0 to 6 . For older participants ( $\geq 70$ years), a value of 1 was added to the total score to correct for age. 
Participants with a total score $\geq 3$ were considered nutritionally at risk.

Sex, age, current medical conditions, current symptoms, unintentional weight loss, eating and drinking habits, living and social situation, daily activity, medications and illicit drug use, smoking, alcohol intake, and primary diagnosis were recorded. A complete physical examination was performed, and blood pressure, heart rate, and body temperature were reported. Anthropometric data included weight (nearest $0.1 \mathrm{~kg}$ ), height (nearest $0.01 \mathrm{~m}$ ), BMI (calculated as weight/height $^{2}$ ), skin-fold thickness (triceps, biceps, back, abdomen, and thigh; nearest $1 \mathrm{~mm}$ ), and upper arm circumference (nearest $0.1 \mathrm{~cm}$ ). All skin-fold thickness measurements were performed three times (serially) by one examiner with a Lange ${ }^{\circledR}$ caliper, using the mean value of the three measurements. Routine laboratory tests were performed, including measurement of albumin, prealbumin, and transferrin.

After values were obtained for the triceps skin-fold (= TSF in $\mathrm{mm}$ ) and the mid-arm circumference (= MAC in $\mathrm{cm})$, the mid-arm muscle area (= MAMA in $\mathrm{cm}^{2}$ ) was calculated using the corrected formula described by Heymsfield et al. [25]:

For men: $\left[(\mathrm{MAC}-\pi \times \mathrm{TSF})^{2}: 4 \pi\right]-10$

For women: $\left[(\mathrm{MAC}-\pi \times \mathrm{TSF})^{2}: 4 \pi\right]-6.5$

A bioimpedance analysis (BIA) was performed for every patient using the Bioimpedance Analyzer Model $\mathrm{STA}^{\circledR}$, Soft Tissue-analyzer (Akern Bioresearch, Florence, Italy) with an operating frequency of $50 \mathrm{kHz}$ at $800 \mathrm{~mA}$ and standard electrode locations on the right hand and foot. Phase angle was taken as one measurement, incorporating information on reactance and impedance. Whole-body BIA measurements were taken with the subject in a supine position on a non-conducting surface, with the arms slightly abducted from the trunk and the legs slightly separated, as previously described [26]. All patients were measured by the same two members of the hospital staff. The software used was BC3GERM version 1.4 (Akern/ Fresenius, Bad Homburg, Germany). In each patient, we measured the fat-free mass, the body fat, the body cell mass, the total body water, the extracellular water, and the intracellular water. For body cell mass calculation, the formula of Roza et al. was used [27]. For lean body mass calculation, the formula of Moore et al. was used [28].

\section{Statistical analysis}

Means and proportions of variables were calculated for participant characteristics. Bivariate analysis was used to compare prevalence of glucocorticoid treatment, immobility, and constipation in patients at nutritional risk (NRS$2002 \geq 3$ ) and not at risk (NRS-2002 < 3). In a first step, continuous data were analyzed by QQplots to test for normal distribution of the sample. If data were normally distributed, a parametric two-sided test such as Student's $t$-test was used; if data distribution was abnormal, Wilcoxon signed-rank tests were used. For binary data, chi-square tests were used. To reduce the possibility of confounders, the effects and their magnitude were analyzed in a multiple logistic regression model in stepwise fashion. Nutritional risk defined by an NRS-2002 score $\geq 3$ was used as dependent variable. To test whether a factor was a possible confounder (i.e., age, height, lean body mass, transferrin, and diagnosis of malignancy) or had an importance in association with nutritional risk, likelihood ratio tests were performed between the different logistic regression models. To test for potential interactions between factors of the logistic regression models, we used the respective interaction terms of our statistical software. In a possible case of collinearity, Mantel-Haenszel analysis was used and statistical significance of the interaction was then determined using the Breslow-Day test of homogeneity.

Weight and BMI were not tested for possible colinearity, because by definition, the NRS-2002 includes weight loss. A $p$ value (two-sided) $<0.05$ was considered as statistically significant. Data analysis was performed with STATA $^{\circledR}$ for Windows, version 6.0 (StataCorp LP, College Station, Texas, USA).

\section{Results}

One hundred and fifty-one patients were admitted to the general medical wards during January 2004, 101 of whom were eligible for and agreed to participate in the study. Participant characteristics are shown in Table 1, divided into patients at nutritional risk (NRS-2002 $\geq 3$ ) or not at risk (NRS-2002 <3). Participants who were excluded were not significantly different from the included participants with regard to age, gender, or clinical and laboratory findings; thus, our study collective is representative of the patients on the general medical wards of our university hospital (data not shown).

To check whether characteristics of patients at nutritional risk have changed since 2004, data from our quality control database of February 2008 were taken to compare prevalence of patients at nutritional risk in our department of internal medicine [29]. The prevalence was 53\% in 2008 and was not significantly different from the data set presented here. Nutritional risk, defined by an NRS-2002 total score $\geq 3$, was identified in $48 \%$ of participants.

Sixteen out of 101 patients were on glucocorticoid treatment for ulcerous colitis, chronic obstructive pulmonary disease, or malignancy. The groups in 2004 and 2008 did not significantly differ in age, polypharmacy, or 
Table 1 Bivariate analysis of factors associated with nutritional risk
$S D$ standard deviation, NRS $2002<3$ not at nutritional risk, $N R S-2002 \geq 3$ at nutritional risk, $n$ number of patients

Asterisks indicate that $p$ values are presented in two decimal places

\begin{tabular}{|c|c|c|c|}
\hline Characteristics (mean \pm SD) & NRS-2002 <3 & NRS-2002 $\geq 3$ & $p$ \\
\hline Age (years) & $55.4 \pm 17.5$ & $64.1 \pm 17.1$ & 0.01 \\
\hline Sex (total, counts) & 48 & 53 & 0.21 \\
\hline Males & 32 & 23 & \\
\hline Females & 21 & 25 & \\
\hline Height (cm) & $170.5 \pm 10.8$ & $164.4 \pm 9.3$ & 0.01 \\
\hline Weight (kg) & $74.7 \pm 16.8$ & $60.3 \pm 14.8$ & 0.01 \\
\hline Body mass index $\left(\mathrm{kg} / \mathrm{m}^{2}\right)$ & $25.7 \pm 5.4$ & $22.2 \pm 4.5$ & 0.01 \\
\hline Triceps skin-fold (mm) & $11.1 \pm 6.2$ & $9.1 \pm 5.1$ & 0.09 \\
\hline Mid-arm circumference $(\mathrm{cm})$ & $25.6 \pm 3.5$ & $22.5 \pm 2.8$ & 0.01 \\
\hline \multicolumn{4}{|l|}{ Mid-arm muscle area $\left(\mathrm{cm}^{2}\right)$ (total) } \\
\hline Males & $47.4 \pm 14.1$ & $36.3 \pm 8.6$ & 0.01 \\
\hline Females & $31.1 \pm 16.3$ & $26.3 \pm 5.9$ & 0.18 \\
\hline Lean body mass (\%) & $53.1 \pm 10.7$ & $43.8 \pm 10.0$ & 0.01 \\
\hline Fat mass $(\%)$ & $21.7 \pm 11.3$ & $16.6 \pm 8.1$ & 0.01 \\
\hline Albumin $(\mathrm{g} / \mathrm{L})$ & $36.7 \pm 7.1$ & $31.6 \pm 5.9$ & 0.01 \\
\hline Prealbumin $(\mathrm{g} / \mathrm{L})$ & $0.21 \pm 0.08$ & $0.20 \pm 0.09$ & 0.70 \\
\hline Transferrin $(\mathrm{g} / \mathrm{L})$ & $1.97 \pm 0.47$ & $1.73 \pm 0.40$ & 0.01 \\
\hline Main diagnosis group $(n)$ & NRS-2002 < $3(\%)$ & NRS-2002 $\geq 3(\%)$ & \\
\hline Cardiovascular disease $(n=39)$ & 39.6 & $37.5 *$ & 0.83 \\
\hline Thoracopulmonal disease $(n=34)$ & 32.1 & $35.4 *$ & 0.72 \\
\hline Abdominal disease $(n=19)$ & 18.9 & $18.8 *$ & 0.99 \\
\hline Malignancy $(n=32)$ & 18.9 & $45.8 * *$ & 0.01 \\
\hline Endocrinological disease $(n=22)$ & 14.5 & $18.8^{*}$ & 0.48 \\
\hline Neurological disease $(n=21)$ & 28.3 & $12.5^{*}$ & 0.05 \\
\hline Skeletal disease $(n=6)$ & 5.6 & $6.2 *$ & 0.90 \\
\hline Glucocorticoids & 7.5 & 25 & 0.02 \\
\hline Constipation & 34.0 & 52.1 & 0.07 \\
\hline Immobility & 26.6 & 45.8 & 0.04 \\
\hline
\end{tabular}

the number of diagnoses. In 2004, the prevalence of glucocorticoid treatment (daily prednisone use $>7.5 \mathrm{mg}$ ) was $25 \%$ in patients at nutritional risk, compared to $8 \%$ in patients not at risk $(p=0.02)$. Similarly, $46 \%$ of the patients at risk were immobile, compared to $27 \%$ of the patients not at risk, a statistically significant difference $(p=0.04)$. Finally, $52 \%$ of the patients at nutritional risk had constipation, compared to only $34 \%$ of the population not at risk $(p=0.07)$, a difference of nearly $20 \%$. These results are shown in Fig. 1.

Patients at risk with NRS-2002 $\geq 3$ showed a reduced MAMA of $40.7 \pm 1.5 \mathrm{~cm}^{2}$, versus $53.1 \pm 2.1 \mathrm{~cm}^{2}$ in patients not at risk $(p<0.001)$. We included possible confounding factors in the model such as malignancy, age, height, and lean body mass. None of these factors were significant or had an influence in the logistic statistical multivariate model (Table 2). In the multivariate model, glucocorticoid treatment was strongly associated with a state of nutritional risk, OR 8.2 (95\% CI 1.7-39.2). Immobility (OR 3.2 [95\% CI 1.2-8.6]) and constipation
(OR 2.3 [95\% CI 0.9-5.8]) were associated with nutritional risk, whereas MAMA and female sex were inversely associated with nutritional risk, respectively. In general, no interaction was found between the factors in the multivariate model. However, a possible interaction of constipation in patients under glucocorticoid treatment associated with nutritional risk could not be completely excluded. Mantel-Haenszel analysis and Breslow test showed a $p$ value of 0.18 .

\section{Discussion}

Sarcopenia is a term that describes the connection between muscle loss, risk of malnutrition, and immobility, which again induces neuromuscular impairment. Whitehead and Lunn [11] first proposed an association between hypercortisolism and malnutrition. The aim of our study was to test the hypothesis that glucocorticoid use and immobility are associated with nutritional risk. Because data exist that 


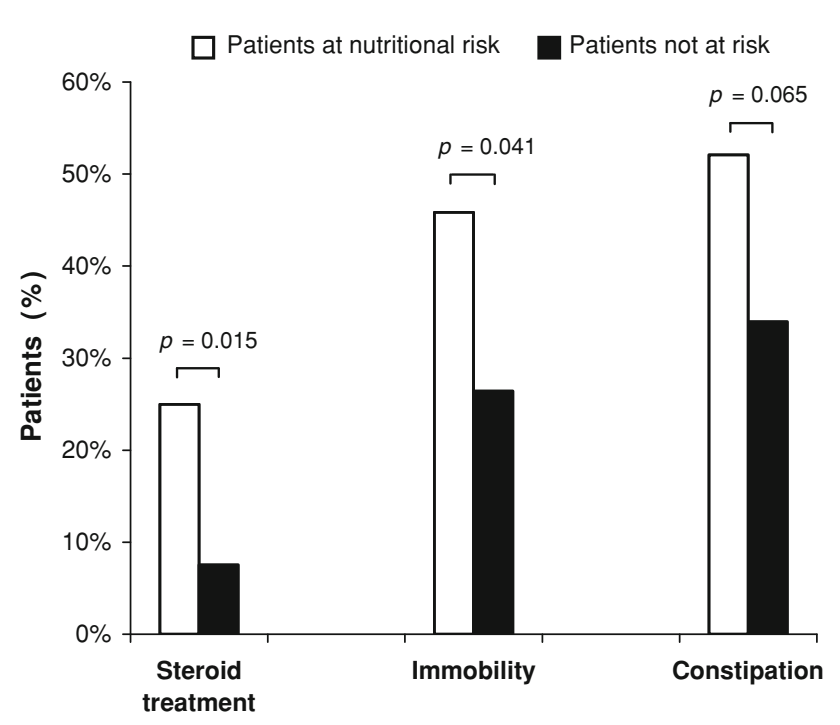

Fig. 1 Univariate analysis demonstrates a predominance of steroid treatment, immobility, and constipation in patients at nutritional risk versus patients not at risk (prevalence of steroid treatment: $25 \%$ versus $8 \%$; prevalence of immobility: $46 \%$ versus $26 \%$; prevalence of constipation: $52 \%$ versus $34 \%$ )

show an association between physical activity and constipation, we additionally investigated whether constipation could be a cofactor associated with malnutrition.

The results of our study confirmed that glucocorticoid use and immobility are independently associated with a risk of malnutrition determined by the NRS-2002. To our surprise, constipation seems to have an association with nutritional risk. In addition, we found, not surprisingly, that middle-arm muscle area was reduced in patients at nutritional risk. Chronic glucocorticoid use may result in reduction in muscle mass, an effect demonstrated by a decrease in middle-arm muscle mass as a result of glucocorticoid-induced catabolism. The reduced muscle mass leads to reduced mobility and a higher risk of falls, which ultimately confines a patient to his bed. To be bedridden is a further risk factor for catabolism, which, in addition to glucocorticoid treatment, is represented by a significant odds ratio in association with an NRS-2002 $\geq 3$. However, because the design of this study is cross-sectional, it is not possible to determine whether glucocorticoids themselves lead to a higher risk of malnutrition. Several points favor the hypothesis that glucocorticoid treatment leads to nutritional risk and muscle wasting.

\section{Glucocorticoids}

Glucocorticoids have a proteolytic effect, which in and of itself can contribute to nutritional risk. In inflammatory bowel disease, there are hints that the risk of malnutrition may be related to an accumulated glucocorticoid dose. Bernal et al. [30] found that the clinical failure to respond to systemic glucocorticoids in active colitis was associated with the presence of nutritional risk. Another study from Geerling et al. [31] showed a variety of nutritional and functional deficiencies in patients with long-standing Crohn's disease in remission, especially those with a high lifetime prednisone dose. Furthermore, it is well known that Cushing's syndrome is accompanied by muscular atrophy of the proximal extremities. These examples from the literature on glucocorticoids support our hypothesis drawn from the multiple logistic regression equation presented in Table 2.

\section{Immobility}

Another factor associated with high nutritional risk in our study was immobility. Immobile patients were defined as patients confined to their bed due to illness and unable to get up for use of the toilet. It is not surprising that immobility is a strong contributor to nutritional risk. Fortyfive percent of our patients with nutritional risk were on bed rest, compared to $25 \%$ of the population not at risk. In the bivariate analysis, immobility alone is statistically significant, with a $p$ value of 0.04 for patients with an NRS$2002 \geq 3$. When the factor "immobility" was analyzed along with the factor "glucocorticoid treatment" in a multivariate model, the association of glucocorticoid use and nutritional risk did not change in a relevant fashion [OR 4.59 (95\% CI: 1.31-16.05, with immobility) from OR 4.54 (95\% CI: 1.32-15.66, without immobility)]. Thus, immobility is an independent predictor of nutritional risk and has no interactive effect on the association between glucocorticoid use and nutritional risk.

This statistical effect was previously observed by Ferrando and co-workers in an experimental study with human volunteers [17]: they found that bed rest had an amplifying effect on the catabolic response of skeletal

Table 2 Odds ratios (OR) and 95\% confidence intervals (CI) of the factors obtained by multiple logistic regression analysis for all patients at nutritional risk $(n=100$, NRS-2002 $\geq 3 ; 1$ observation omitted due to missing values)

\begin{tabular}{lccccc}
\hline & OR & SE & $z$ value & $p$ value & $95 \%$ CI \\
\hline Glucocorticoid use & 8.16 & 6.54 & 2.62 & 0.0096 & $1.70-39.24$ \\
Immobility & 3.23 & 1.64 & 2.312 & 0.021 & $1.194-8.76$ \\
Constipation & 2.27 & 1.08 & 1.73 & 0.084 & $0.90-5.79$ \\
Mid-arm muscle area & 0.92 & 0.26 & -2.85 & 0.004 & $0.87-0.97$ \\
Gender & 0.71 & 0.44 & -0.55 & 0.580 & $0.21-2.39$ \\
\hline
\end{tabular}

Units of measurement yes/no, Immobility yes/no, Constipation yes/ no, Mid-arm muscle are in $\mathrm{cm}^{2}$, Gender female/male

The model was adjusted for age $(p=0.48)$, height $(p=0.37)$, and lean body mass $(p=0.06)$

$S D$ standard deviation, $L R C h i^{2} 31.65$, Prob $>C h i^{2} 0.000$, Pseudo- $R^{2}$ 0.229 , Log likelihood -53.41 
muscle to an infusion of hydrocortisone succinate, determined by phenylalanine kinetics. The authors demonstrated experimentally that absence of muscular activity sensitizes skeletal muscle to the catabolic effects of cortisol. These findings are reflected in the data from our study as well.

\section{Constipation}

Of the initially proposed gastrointestinal symptoms evaluated, only constipation was associated with an NRS-2002 $\geq 3$; nausea and diarrhea did not show any association. Constipation was defined as having a bowel movement fewer than three times per week and stools that were usually hard, dry, small in size, and difficult to eliminate. In the bivariate analysis, we could only find a trend and not a statistically significant association between constipation and risk of malnutrition $(p=0.07)$. Because the prevalence of constipation in the population at nutritional risk was over $30 \%$ and clearly above the prevalence in the general population (15\%), we included constipation in the multivariate model to avoid a type II error. In the model that included glucocorticoid use, immobility, and constipation alone (data not shown), constipation was an independent statistically significant factor $(p=0.044)$, with an OR of 2.41. After inclusion of middle-arm muscle area in the model, constipation was identified as an independent risk factor for nutritional risk with a tendency to be statistically significant. In addition, a possible interaction of constipation with glucocorticoid use and to be at risk of malnutrition could not be excluded by our data, a possible reason for that could be lack of study power. We suggest that muscle atrophy affects not only skeletal muscle fibers but also smooth muscle fibers. This might be an explanation for slower gastrointestinal transit.

\section{Mid-arm muscle area}

Not surprisingly, the mean MAMA was reduced by more than $20 \%$ in the population at nutritional risk compared to the patients not at risk. This factor was negatively correlated with the nutritional risk in the multivariate model (Table 2).

The 95\% CI for age of our study population was 56-63 years, with a range of $18-89$ years. Thus, according to our data, our model for risk of malnutrition and therefore frailty and sarcopenia can be applied not only to a geriatric population but also to a much younger population.

\section{Conclusion}

Glucocorticoid treatment, immobility, and constipation are associated with nutritional risk as defined by the NRS-2002 in a mixed hospitalized population. The presence of constipation, long-time glucocorticoid use, or immobility should alert the clinician to check for nutritional status, which is an important factor in mortality and morbidity.

Acknowledgments The study was supported by the research funds of the Bern University Hospital.

Conflict of interest None of the authors has a conflict of interest to declare.

\section{References}

1. Gallagher-Allred CR, Voss AC, Finn SC, McCamish MA (1996) Malnutrition and clinical outcomes: the case for medical nutrition therapy. J Am Diet Assoc 96:361-366

2. Allison S (2000) Malnutrition, disease, and outcome. Nutrition 16:590-593

3. Correia M, Waitzberg D (2003) The impact of malnutrition on morbidity, mortality, length of hospital stay and costs evaluated through a multivariate model analysis. Clin Nutr 22:235-239

4. McWhirter J, Pennington C (1994) Incidence and recognition of malnutrition in hospital. BMJ 308:945-948

5. Pirlich M, Schütz T, Noramn K, Gastell S, Lübke HJ, Bischoff SC, Bolder U, Frieling T, Güldenzoph H, Hahn K, Jauch KW, Schindler K, Stein J, Volkert D, Weimann A, Werner H, Wolf C, Zürcher G, Bauer P, Lochs H (2006) The German hospital malnutrition study. Clin Nutr 25:563-572

6. Norman K, Pichard C, Lochs H, Pirlich M (2007) Prognostic impact of disease-related malnutrition. Clin Nutr 27:5-15

7. Sorensen J, Kondrup J, Prokopowicz J, Schiesser M, Krahenbuhl L, Meier R, Liberda M (2008) EuroOOPS: an international, multicentre study to implement nutritional risk screening and evaluate clinical outcome. Clin Nutr 27:340-349

8. Kondrup J, Allison S, Elia M, Vellas B, Plauth M (2003) ESPEN guidelines for nutrition screening 2002. Clin Nutr 22:415-421

9. Schwegler I, von Holzen A, Gutzwiller JP, Schlumpf R, Mühlebach S, Stanga Z (2010) Nutritional risk is a clinical predictor of postoperative mortality and morbidity in patients undergoing surgery for colorectal cancer. Brit J Surg 97:92-97

10. Muhlberg W, Sieber C (2004) Sarcopenia and frailty in geriatric patients: implications for training and prevention. Z Gerontol Geriatr 37:2-8

11. Whitehead RG, Lunn PG (1979) Endocrines in protein-energy malnutrition. Proc Nutr Soc 38:69-76

12. Manary MJ, Muglia LJ, Vogt SK, Yarashski KE (2006) Cortisol and its action on the glucocorticoid receptor in malnutrition and acute infection. Metab Clin Exp 55:550-554

13. Lunn PG, Whitehead RG, Hay RW, Baker BA (1973) Progressive changes in serum cortisol, insulin and growth hormone concentrations and their relationship to the distorted amino acid pattern during the development of kwashiorkor. Br J Nutr 29:399-422

14. Lunn PG, Whitehead RG, Baker BA, Austin S (1976) The effect of cortisone acetate on the course of development of experimental protein-energy malnutrition in rats. Br J Nutr 36:537-550

15. Wolfe RR (2006) The underappreciated role of muscle in health and disease. Am J Clin Nutr 84:475-482

16. Langhans W (2002) Peripheral mechanisms involved with catabolism. Curr Opin Clin Nutr Metab Care 5:419-426

17. Ferrando AA, Stuart CA, Sheffield-Moore M, Wolfe RR (1999) Inactivity amplifies the catabolic response of skeletal muscle to cortisol. J Clin Endocrinol Metab 84:3515-3521 
18. Pirlich M, Biering H, Gerl H, Ventz M, Schmidt B, Ertl S, Lochs $\mathrm{H}$ (2002) Loss of body cell mass in Cushing's syndrome: effect of treatment. J Clin Endocrinol Metab 87:1078-1084

19. Lewis JD, Gelfand JM, Troxel AB, Forde KA, Newcomb C, Kim H, Margolis DJ, Strom BL (2008) Immunosuppressant medications and mortality in inflammatory bowel disease. Am J Gastroenterol 103:1428-1435

20. Ewig S, Birkner N, Strauss R, Schaefer E, Pauletzki J, Bischoff H, Schraeder P, Welte T, Hoeffken G (2009) New perspectives on community-acquired pneumonia in 338406 patients. Results from a nationwide mandatory performance measurement programme in healthcare quality. Thorax 64:1062-1069

21. Kinnunen O (1991) Study of constipation in a geriatric hospital, day-hospital, old people's home and at home. Aging 3:161-170

22. Müller-Lissner SA, Kamm MA, Scarpignato C, Wald A (2005) Myths and misconceptions about chronic constipation. Am J Gastroenterol 100:232-242

23. Kondrup J, Rasmussen HH, Hamberg O, Stanga Z (2003) Nutritional risk screening (NRS 2002): a new method based on an analysis of controlled clinical trials. Clin Nutr 22:321-336

24. Olin AO, Osterberg P, Hådell K, Armyr I, Jerström S, Ljungqvist O (1996) Energy-enriched hospital food to improve energy intake in elderly patients. J Parenter Enteral Nutr 20:93-97
25. Heymsfield SB, McManus C, Smith J, Stevens V, Nixon DW (1982) Anthropometric measurement of muscle mass: revised equations for calculating bone-free arm muscle area. Am J Clin Nutr 36:680-690

26. Kushner RF, Schoeller DA (1986) Estimation of total body water by bioelectrical impedance analysis. Am J Clin Nutr 44:417-424

27. Roza AM, Shizgal HM (1984) The Harris Benedict equation reevaluated: resting energy requirements and the body cell mass. Am J Clin Nutr 40:168-182

28. Moore F, Oleson K, McMurphy J, Parker H, Bell M, Boyden C (1963) The body cell mass and its supporting environment. Body composition in health and disease. Saunders \& Co, Philadelphia

29. Iff S, Leuenberger M, Sterchi AB, Stanga Z (2008) Nutritional management study: screening part. Clin Nutr 3:154 (abstract)

30. Bernal I, Manosa M, Domenech E, Garcia-Planella E, Navarro M, Lorenzo-Zuniga V, Cabre E, Gassull MA (2006) Predictors of clinical response to systemic steroids in active ulcerative colitis. Dig Dis Sci 51:1434-1438

31. Geerling BJ, Badart-Smook A, Stockbrugger RW, Brummer RJ (1998) Comprehensive nutritional status in patients with longstanding Crohn disease currently in remission. Am J Clin Nutr 67:919-926 